 \\ Journal of \\ Human, Earth, and Future
}

ISSN: 2785-2997

Vol. 2, No. 3, September, 2021

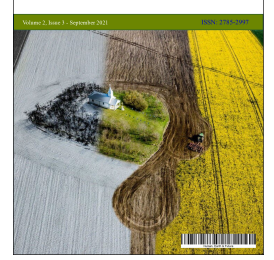

\section{Analytical Solution of Unsteady-state Forchheimer Flow Problem in an Infinite Reservoir: The Boltzmann Transform Approach}

\author{
Temitayo Sheriff Adeyemi ${ }^{1 *} \odot$ \\ ${ }^{1}$ Department of Petroleum Engineering, University of Ibadan, Ibadan, Nigeria
}

Received 04 June 2021; Revised 09 August 2021; Accepted 16 August 2021; Published 01 September 2021

\begin{abstract}
For several decades, attempts have been made by several authors to develop models suitable for predicting the effects of Forchheimer flow on pressure transients in porous media. However, due to the complexity of the problem, they employed numerical and/or semi-analytical approaches, which greatly affected the accuracy and range of applicability of their results. Therefore, in order to increase accuracy and range of applicability, a purely analytical approach to solving this problem has been introduced and applied. Therefore, the objective of this paper is to develop a mathematical model suitable for quantifying the effects of turbulence on pressure transients in porous media by employing a purely analytical approach. The partial differential equation (PDE) that governs the unsteady-state flow in porous media under turbulent conditions is obtained by combining the Forchheimer equation with the continuity equation and equations of state. The obtained partial differential equation (PDE) is then presented in dimensionless form (by defining appropriate dimensionless variables) in order to enhance more generalization in application, and the method of Boltzmann Transform is employed to obtain an exact analytical solution of the dimensionless equation. Finally, the logarithmic approximation (for larger times) of the analytical solution is derived. Moreover, after rigorous mathematical modeling and analysis, a novel mathematical relationship between dimensionless time, dimensionless pressure, and dimensionless radius was obtained for an infinite reservoir dominated by turbulent flow. It was observed that this mathematical relationship bears some similarities with that of unsteady-state flow under laminar conditions. Their logarithmic approximations also share some similarities. In addition, the results obtained show the efficiency and accuracy of the Boltzmann Transform approach in solving this kind of complex problem.
\end{abstract}

Keywords: Analytical Solution; Unsteady-state; Forchheimer Flow; Boltzmann Transform; Infinite Reservoirs.

\section{Introduction}

Darcy's equation (which assumes a linear relationship between pressure gradient and velocity) is one of the fundamental equations of porous media flow. However, Darcy's equation is only valid for viscous (laminar) flow. This is due to the fact that in turbulent flow, the relationship between pressure gradient and velocity is not linear. The Forchheimer equation emerged as an attempt to model the departure from Darcy's law at high velocity. The Forchheimer equation is given as:

$-\frac{\mathrm{dp}}{\mathrm{dr}}=\frac{\mu}{\mathrm{K}} \mathrm{v}+\beta \rho \mathrm{v}^{2}$

* Corresponding author: spegenius@gmail.com

doi) http://dx.doi.org/10. 28991/HEF-2021-02-03-04

$>$ This is an open access article under the CC-BY license (https://creativecommons.org/licenses/by/4.0/).

(C) Authors retain all copyrights. 
The above equation governs non-Darcy flow in porous media. Attempts have been made by several authors to establish the validity of the Forchheimer equation and also demonstrate its applicability to porous media flow. Geertsma (1974) developed a relationship between the Forchheimer coefficient and rock properties and also validated the equation with experimental data [1]. Hassanizadeh \& Gray (1987) provided the physical basis for the Forchheimer equation and identified the source of the non-linearity [2]. Giorgi (1997) derived the Forchheimer equation using matched asymptotic expansion [3].

Zimmerman et al. (2004) confirmed the existence of a weak inertia regime and showed that the Forchheimer equation can probably be used over the entire range of Reynolds numbers [4]. Fourar et al. (2004) analyzed the effect of space dimensions on the development of flow regimes [5]. Lucas et al. (2007) presented that quadratic deviation in Forchheimer's law appears as a result of non-periodicity in porous media and fractures [6]. Several other authors have modelled the Forchheimer flow in porous media by employing the numerical method [7-13], and semi-analytical approach [14-25].

The objective of this paper is to develop a mathematical model suitable for quantifying the effects of turbulence on pressure transients in porous media by employing a purely analytical approach. The partial differential equation (PDE) that governs the unsteady-state flow in porous media under turbulent condition is obtained by combining the Forchheimer equation with the continuity equation and equations of state. The obtained partial differential equation (PDE) is then presented in dimensionless form (by defining appropriate dimensionless variables) in order to enhance more generalization in application, and the method of Boltzmann Transform is employed to obtain an exact analytical solution of the dimensionless equation. Finally, the logarithmic approximation (for larger times) of the analytical solution is derived. Moreover, after a rigorous mathematical modeling and analysis, a novel mathematical relationship between dimensionless time, dimensionless pressure, and dimensionless radius was obtained for an infinite reservoir dominated by turbulent flow.

\section{Research Methodology}

The flow chart below shows the processes involved in proffering a solution to the unsteady-state Forchheimer flow problem in an infinite reservoir by employing the Boltzmann Transform Approach.
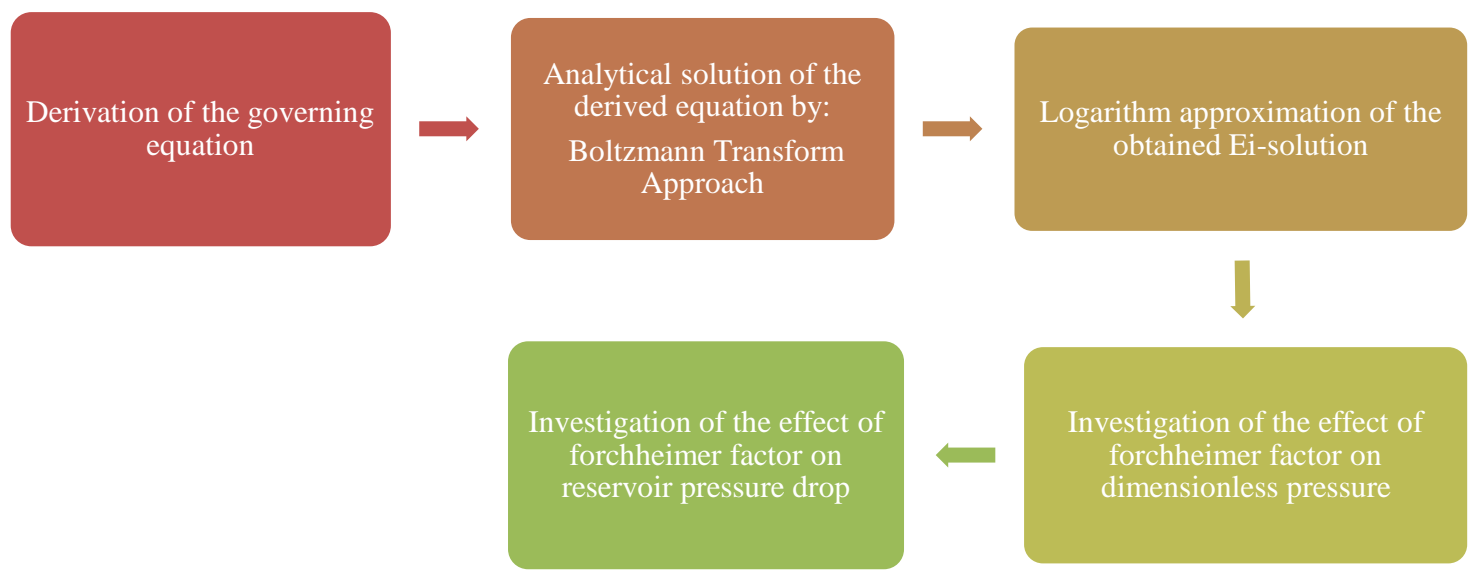

Figure 1. Flowchart of the research methodology

\subsection{Mathematical Development}

In this section, the equation that governs Forchheimer flow in porous media under unsteady-state conditions is derived. This equation is also presented in dimensionless form by defining the appropriate dimensionless variables in order to ensure a more generalized application.

\subsubsection{Mathematical Modeling of Forchheimer Flow}

The mathematical modeling of fluids transport in porous media involves the combination of three categories of equations, namely; the continuity equation, transport equation and the equations of state.

The continuity equation for radial flow in porous media is given as:

$$
-\frac{1}{\mathrm{r}} \frac{\partial}{\partial \mathrm{r}}(e \mathrm{vr})=\frac{\partial}{\partial \mathrm{t}}(\mathrm{e} \phi)
$$

For the case of Forchheimer flow, the transport equation is the Forchheimer equation and it is given as: 
$-\frac{\partial \mathrm{p}}{\partial \mathrm{r}}=\frac{\mu \mathrm{V}}{\mathrm{K}}+\beta \mathrm{ev}^{2}$

Finally, the set of equations of state for fluid and formation compressibility as given as follow:

$c=-\frac{1}{v} \frac{\partial v}{\partial p}$

$c=\frac{1}{e} \frac{\partial e}{\partial p}$

$C_{f}=\frac{1}{\phi} \frac{\partial \phi}{\partial p}$

Equations 3(a) and 3(b) describe fluids compressibility while Equation 3(c) is the equations for rock formation (porous media) compressibility. In order to obtain the mathematical expression for Forchheimer flow under unsteadystate condition, Equations 1 to 3 must be combined. Expanding the right-hand-side (RHS) of the Equation 1, we have:

$-\frac{1}{r} \frac{\partial}{\partial r}(e v r)=e \frac{\partial \phi}{\partial t}+\phi \frac{\partial e}{\partial t}$

$-\frac{1}{r} \frac{\partial}{\partial r}(e v r)=e \frac{\partial \phi}{\partial t} \cdot \frac{\partial p}{\partial t}+\phi \frac{\partial e}{\partial p} \cdot \frac{\partial p}{\partial t}$

$-\frac{1}{r} \frac{\partial}{\partial r}(e v r)=e \phi\left[\frac{1}{\phi} \frac{\partial \phi}{\partial p} \cdot \frac{\partial p}{\partial t}+\frac{1}{e} \frac{\partial e}{\partial p} \cdot \frac{\partial p}{\partial t}\right]$

Substituting Equations 3(b) and 3(c) into the above equation yields:

$-\frac{1}{r} \frac{\partial}{\partial \mathrm{r}}(e v r)=e \phi\left(C_{f}+C\right) \frac{\partial p}{\partial t}$

Simplifying further, we have:

$-\frac{1}{r} \frac{\partial}{\partial \mathrm{r}}(\mathrm{evr})=\phi C_{t} \frac{\partial p}{\partial t}$

Where $C_{t}=C+C_{f}$

Solving Equation 2 for $v$, we have:

$-\frac{\partial \mathrm{p}}{\partial \mathrm{r}}=\frac{\mu}{K} v+\beta e v^{2}$

$-\frac{k}{\mu} \frac{\partial \mathrm{p}}{\partial \mathrm{r}}=v+\frac{\beta e k v^{2}}{\mu}$

$\mathrm{v}=-\frac{F k}{\mu} \frac{\partial p}{\partial r}$

where $\mathrm{F}$ is the parameter that accounts for the departure from Darcy's viscous flow.

Substituting Equation 6 into 5 we have:

$\frac{1}{r} \frac{\partial}{\partial \mathrm{r}}\left(\frac{r F k}{\mu} \frac{\partial p}{\partial r}\right)=\phi C_{t} \frac{\partial p}{\partial t}$

Expanding the above equations and simplifying further, we obtain the following equation

$\frac{\partial^{2} p}{\partial r^{2}}+\frac{1}{r} \frac{\partial \mathrm{p}}{\partial \mathrm{r}}=\frac{\phi \mu C_{t}}{k F} \frac{\partial p}{\partial t}$

The above equation governs Non-Darcy flow in porous media under unsteady-state condition.

\subsubsection{Dimensionless Transformation}

Presenting equations in dimensionless form has always been of great advantage in Science and Engineering as it makes results to be applicable on a more global scale. By defining the following dimensionless variables;

$\mathrm{t}_{\mathrm{D}}=\frac{2.637 * 10^{-4} \mathrm{kt}}{\phi \mu \mathrm{c}_{\mathrm{t}} \mathrm{r}_{\mathrm{w}}^{2}}$

$\mathrm{r}_{\mathrm{D}}=\frac{\mathrm{r}}{\mathrm{r}_{\mathrm{w}}}$

$\mathrm{P}_{\mathrm{D}}=\frac{7.08 * 10^{-3} \mathrm{KFh}\left(\mathrm{P}_{i}-\mathrm{P}\right)}{\mathrm{q} \mu \mathrm{B}}$

Applying Equations 9(a) to 9(c) on Equation 8, we have:

$\frac{\partial^{2} P_{D}}{\partial r_{D}{ }^{2}}+\frac{1}{r_{D}} \frac{\partial P_{D}}{\partial r_{D}}=\frac{1}{F} \frac{\partial P_{D}}{\partial t_{D}}$

Equation 10 above is the equation for Forchheimer flow in porous media under transient condition. For the case of constant rate production, the associated Initial and Boundary conditions are as follows: 
$\left.\begin{array}{l}\mathrm{P}_{\mathrm{D}}\left(\mathrm{r}_{\mathrm{D}}, \mathrm{t}_{\mathrm{D}}=0\right)=0 \\ \left.\mathrm{r}_{\mathrm{D}} \frac{\partial \mathrm{P}_{\mathrm{D}}}{\partial \mathrm{r}_{\mathrm{D}}}\right]_{\mathrm{r}_{\mathrm{D}}=1}=-1 \\ \mathrm{P}_{\mathrm{D}}\left(\mathrm{r}_{\mathrm{D}} \rightarrow \infty, \mathrm{t}_{\mathrm{D}}\right)=0\end{array}\right\}$

\section{Analytical Solution}

In the previous section, the following mathematical problem was developed:

$\frac{\partial^{2} P_{D}}{\partial r_{D}^{2}}+\frac{1}{r_{D}} \frac{\partial P_{D}}{\partial r_{D}}=\frac{1}{F} \frac{\partial P_{D}}{\partial t_{D}}$

$P_{D}\left(r_{D}, t_{D}=0\right)=0$

$\left[r_{D} \frac{\partial P_{D}}{\partial r_{D}}\right]_{r_{D}=1}=-1$

$P_{D}\left(r_{D} \rightarrow \infty, t_{D}\right)=0$

In this section, the method of Boltzmann transformation is employed to obtain the exact analytical solution of the above problem.

\subsection{Boltzmann's Transformation}

In this section, the mathematical problem above is transformed into an ordinary differential equation that is easier to solve via Boltzmann transform. The Boltzmann transform variable is defined as;

$E_{D}=a r_{D}^{b} t_{D}^{c}$

Combining Equations 10 and 12(a), we have

$\frac{\partial^{2} P_{D}}{\partial \epsilon_{D}}+\left[\frac{1}{\left(\partial \epsilon_{D} / \partial r_{D}\right)^{2}} \frac{\partial^{2} \epsilon_{D}}{\partial r_{D}^{2}}+\frac{1}{r_{D}} \frac{1}{\left(\partial \epsilon_{D} / \partial r_{D}\right)}-\frac{1}{F\left(\partial \epsilon_{D} / \partial r_{D}\right)^{2}} \frac{\partial \epsilon_{D}}{\partial t_{D}}\right] \frac{\partial p_{D}}{\partial \epsilon_{D}}=0$

From Equation 12, we have:

$\frac{\partial \epsilon_{D}}{\partial t_{D}}=a r^{b}{ }_{D} \frac{\partial}{\partial t_{D}}\left(t_{D}{ }^{c}\right)=a r^{b}{ }_{D} t_{D}{ }^{c} \cdot \frac{C}{t_{D}}$

$\therefore \frac{\partial \epsilon_{D}}{\partial r_{D}}=\frac{C}{t_{D}} \in_{D}$

$\frac{\partial \epsilon_{D}}{\partial r_{D}}=\frac{\partial}{\partial r_{D}}\left(r_{D}^{b}\right) \cdot a t_{D}^{c}=a r_{D}^{b} t_{D}^{c} \cdot \frac{b}{r_{D}}$

$\therefore \frac{\partial \epsilon_{D}}{\partial r_{D}}=\frac{b}{r_{D}} \epsilon_{D}$

$\frac{\partial^{2} \in_{D}}{\partial r_{D}{ }^{2}}=\frac{\partial}{\partial r_{D}}\left(a t_{D}{ }^{c} b r_{D}{ }^{b-1}\right)=a t_{D}^{c} \cdot b(b-1) r_{D}{ }^{b-2}$

$\frac{\partial^{2} \epsilon_{D}}{\partial r_{D}{ }^{2}}=a r_{D}{ }^{b} t_{D}^{c} b(b-1) r_{D}{ }^{-2}=\frac{b(b-1)}{r_{D}^{2}} \epsilon_{D}$

The multiplier term in Equation 12(b) is;

$\frac{1}{\left(\partial \epsilon_{D} / \partial r_{D}\right)^{2}} \frac{\partial^{2} \epsilon_{D}}{\partial r_{D}^{2}}+\frac{1}{r_{D}\left(\partial \epsilon_{D} / \partial r_{D}\right)}-\frac{1}{F\left(\partial \epsilon_{D} / \partial r_{D}\right)^{2}} \frac{\partial \epsilon_{D}}{\partial t_{D}}$

Substituting Equations 13(a) to 13(c) into 13(d), we have;

$\frac{1}{\left(b \epsilon_{D} / r_{D}\right)^{2}} \frac{b(b-1)}{r_{D}{ }^{2}} \epsilon_{D}+\frac{1}{r_{D}} \frac{1}{b \epsilon_{D} / r_{D}}-\frac{1}{\left(b \epsilon_{D} / r_{D}\right)^{2}} \frac{C}{F t_{D}} \in_{D}$

The above expression reduces to;

$\frac{b-1}{b} \cdot \frac{1}{\epsilon_{D}}+\frac{1}{b} \frac{1}{\epsilon_{D}}-\frac{c r_{D}{ }^{2}}{F b^{2} t_{D}} \frac{1}{\epsilon_{D}}$

Considering the last term in the above equation, we have:

$-\frac{c r_{D}^{2}}{F b^{2} t_{D}} \cdot \frac{1}{\epsilon_{D}}=-\frac{c r_{D}^{2}}{F b^{2} t_{D}} \frac{1}{a r_{D}^{2} t_{D}{ }^{c}}=-\frac{c}{F} \frac{1}{a b^{2}} \frac{r_{D}^{2}}{r_{D} b} \frac{t_{D}{ }^{-1}}{t_{D}{ }^{C}}$ 
An attempt will be made to determine the value of $\mathrm{a}, \mathrm{b}$ and $\mathrm{c}$ by setting the entire term equal to $\frac{1}{F}$, then eliminating the $r_{D}$ and $t_{D}$ terms by establishing the constant $\mathrm{b}$ and c systematically we have,

$\frac{r_{D}^{2}}{r_{D} b} \equiv 1$ if $b=2$ and $\frac{t_{D}^{-1}}{t_{D}^{c}} \equiv 1$ if $c=-1$

Setting the entire term equal to $\frac{1}{F}$ we have

$\frac{c}{F} \frac{1}{a b^{2}} \frac{r_{D}^{2}}{r_{D}{ }^{b}} \cdot \frac{t_{D}{ }^{-1}}{t_{D}{ }^{c}}=\frac{1}{F}$

Substituting $b=2$ and $c=-1$, we obtain

$a=\frac{1}{4}$

The final form of Equation 13(e) is;

$\frac{2-1}{2} \frac{1}{\epsilon_{D}}+\frac{1}{2} \frac{1}{\epsilon_{D}}+\frac{1}{F}=\left[\frac{1}{\epsilon_{D}}+\frac{1}{F}\right]$

Substituting Equation 13(f) into 12(b), we have;

$\frac{\partial^{2} P_{D}}{\partial \epsilon_{D}^{2}}+\left[\frac{1}{F}+\frac{1}{\epsilon_{D}}\right] \frac{\partial P_{D}}{\partial \epsilon_{D}}=0$

where the following definitions apply:

$\epsilon_{\mathrm{D}}=\operatorname{ar}_{\mathrm{D}} \mathrm{b}_{\mathrm{D}}^{\mathrm{c}}$

$a=\frac{1}{4}, b=2$ and $c=-1$

$\epsilon_{D}=\frac{\mathrm{r}_{\mathrm{D}}^{2}}{4 \mathrm{t}_{D}}$

Equation 14 is the "Boltzmann" transformed form of Equation 10 and Equation 15 is the defined "Boltzmann" variable. In a similar vein, the initial and boundary conditions are transformed as follows;

$\left.P_{D}\left(r_{D}, t_{D} \leq 0\right)=0\right)$

where for $t_{D} \rightarrow 0 ; \in_{D} \rightarrow \infty$, which gives:

$P_{D}\left(\in_{D} \rightarrow \infty\right)=0$

$\left.P_{D}\left(r_{D} \rightarrow \infty, t_{D}\right)=0\right)$

as $r_{D} \rightarrow \infty ; \in_{D} \rightarrow \infty$, which yields

$P_{D}\left(\in_{D} \rightarrow \infty\right)=0$

It can be seen that Equations 16(a) and 16(b) above are the same, this confirms the validity of the Boltzmann transformation for this problem as the "collapsing" of the initial and outer boundary conditions must occur for the Boltzmann transform to be technically valid. Also, transforming the inner boundary condition, we have:

$\left[r_{D} \frac{\partial \epsilon_{D}}{\partial r_{D}} \frac{\partial P_{D}}{\partial \epsilon_{D}}\right]_{r_{D} \rightarrow 0}=\left[r_{D}\left(\frac{1}{r_{D}} \epsilon_{D}\right) \frac{\partial P_{D}}{\partial \epsilon_{D}}\right]_{r_{D} \rightarrow 0, \in_{D} \rightarrow 0}=2\left[\epsilon_{D} \frac{\partial P_{D}}{\partial \epsilon_{D}}\right]_{\epsilon_{D} \rightarrow 0}=-1$

$\therefore\left[\epsilon_{D} \frac{\partial P_{D}}{\partial \epsilon_{D}}\right]_{\in_{D} \rightarrow 0}=-\frac{1}{2}$

\subsection{Solution of the Transformed Problem}

In the previous section, the following transformed form of the mathematical problem developed in section (2) was obtained;

$\frac{\partial^{2} P_{D}}{\partial \epsilon_{D}^{2}}+\left[\frac{1}{F}+\frac{1}{\epsilon_{D}}\right] \frac{\partial P_{D}}{\partial \epsilon_{D}}=0$

$P_{D}\left(\epsilon_{D} \rightarrow \infty\right)$

$\left[\epsilon_{D} \frac{\partial P_{D}}{\partial \epsilon_{D}}\right]=-\frac{1}{2}$

In this section, a solution of the above problem is obtained as follows;

Substituting $V=\frac{\partial P_{\boldsymbol{D}}}{\boldsymbol{\partial} \epsilon_{\boldsymbol{D}}}$ into Equation 14; we have;

$\frac{\partial \mathrm{V}}{\partial \epsilon_{\mathrm{D}}}+\left[\frac{1}{\mathrm{~F}}+\frac{1}{\epsilon_{\mathrm{D}}}\right] \mathrm{V}=0$ 
Solving the above equation, we have:

$\frac{\partial \mathrm{v}}{\partial \epsilon_{\mathrm{D}}}=-\left[\frac{1}{\mathrm{~F}}+\frac{1}{\epsilon_{\mathrm{D}}}\right] \mathrm{v}$

$\int \frac{\partial \mathrm{v}}{\mathrm{V}}=-\int\left(\frac{1}{\mathrm{~F}}+\frac{1}{\epsilon_{\mathrm{D}}}\right) \partial \epsilon_{\mathrm{D}}$

In $V=-\int \frac{1}{F} \mathrm{~d} \epsilon_{\mathrm{D}}-\int \frac{1}{\epsilon_{\mathrm{D}}} \mathrm{d} \epsilon_{\mathrm{D}}$

In $\mathrm{V}=\frac{-\epsilon_{\mathrm{D}}}{\mathrm{F}}-\ln \epsilon_{\mathrm{D}}+\mathrm{K}$

$\mathrm{V}=\frac{\mathrm{C}}{\epsilon_{\mathrm{D}}} e^{\frac{-\epsilon_{\mathrm{D}}}{\mathrm{F}}}$

where $\mathrm{K}$ and $\mathrm{C}$ are constants,

Substituting Equation 16(c) into Equation 17, we have:

$\frac{d P_{D}}{d \epsilon_{D}}=-\frac{1}{2 \epsilon_{D}} e^{\frac{-\epsilon_{\mathrm{D}}}{\mathrm{F}}}$

Integrating the above equation, we obtain the following results;

$F \frac{d P_{D}}{d \epsilon_{D}}=-\frac{1}{2} \frac{\mathrm{F}}{\epsilon_{D}} e^{\frac{-\epsilon_{\mathrm{D}}}{\mathrm{F}}}$

$F \int_{P_{D}=0}^{P_{D}} d P_{D}=-\frac{1}{2} \int_{\epsilon_{D}=\infty}^{\epsilon_{D}} \frac{\mathrm{F}}{\epsilon_{D}} e^{\frac{-\epsilon_{\mathrm{D}}}{\mathrm{F}}} d \epsilon_{D}$

Let $y=\frac{\epsilon_{\mathrm{D}}}{\mathrm{F}}$

$F \int_{P_{D}=0}^{P_{D}} d P_{D}=-\frac{\mathrm{F}}{2} \int_{y=\frac{\mathrm{r}_{\mathrm{D}}{ }^{2}}{4 \mathrm{Ft}_{D}}}^{\infty} \frac{1}{y} e^{-y} d y$

Therefore, we have;

$P_{D}\left(r_{D}, t_{D}\right)=\frac{1}{2} \mathrm{E}_{\mathrm{i}}\left(\frac{\mathrm{r}_{\mathrm{D}}^{2}}{4 \mathrm{Ft}_{D}}\right)$

where $\mathrm{E}_{\mathrm{i}}(x)=\int_{\infty}^{x} \frac{e^{-x}}{x} d x$

The Equation 18 describes the Forchheimer flow in porous media under unsteady-state condition.

\subsubsection{Logarithm Approximation}

Although Equation 18 obtained in the previous section looks very simple, the $\mathrm{E}_{i}$ - function associated with it is computationally rigorous and requires numerical scheme. Therefore, an approximated form of Equation 18 that is valid for large times and does not involve the $\mathrm{E}_{i}$ - function is presented in this section.

When $\frac{\mathrm{r}_{\mathrm{D}}^{2}}{4 \mathrm{Ft}_{D}} \leq 0.01$, we obtain the following form of Equation 18;

$P_{D}\left(r_{D}, t_{D}\right)=\frac{1}{2} \ln \left(\frac{1.781 \mathrm{r}_{\mathrm{D}}^{2}}{4 \mathrm{Ft}_{D}}\right)$

The Equation 19 is another form of Equation 18 that is valid for large times.

Finally, substituting the expressions for $p_{D}, r_{D}$ and $t_{D}$ into Equation 18 and Equation 19, we obtain the following results;

$P=p_{i}-\frac{70.62}{\mathrm{KFh}} \mathrm{E}_{i}\left(\frac{\mathrm{r}_{\mathrm{D}}^{2}}{4 \mathrm{Ft}_{D}}\right)$

$P=p_{i}-\frac{141.2 q \mu B}{\mathrm{KFh}} \ln \left(\frac{1.5 \sqrt{\mathrm{Ft}_{D}}}{\mathrm{r}_{D}}\right)$

Equation 20 describes Forchheimer flow in porous media under unsteady-state condition and Equation 21 is it's logarithm approximation. It is worthy to note that both Equation 20 and Equation 21 are presented in oilfield units.

\section{Results and Discussion}

After a rigorous mathematical analysis and modeling of Forchheimer flow in an infinite reservoir, the results obtained are summarized in mathematical form as follows;

$P_{D}\left(r_{D}, t_{D}\right)=\frac{1}{2} \ln \left(\frac{1.781 \mathrm{r}_{\mathrm{D}}^{2}}{4 \mathrm{Ft}_{D}}\right)$
$P=p_{i}-\frac{70.62}{\mathrm{KFh}} \mathrm{E}_{i}\left(\frac{\mathrm{r}_{\mathrm{D}}^{2}}{4 \mathrm{Ft}_{D}}\right)$ 
$P=p_{i}-\frac{141.2 q \mu B}{\mathrm{KFh}} \ln \left(\frac{1.5 \sqrt{\mathrm{Ft}_{D}}}{\mathrm{r}_{D}}\right)$

Equation 19 above is a mathematical relationship between dimensionless pressure $\left(P_{D}\right)$, dimensionless radius $\left(\mathrm{r}_{\mathrm{D}}\right)$, dimensionless time $\left(t_{D}\right)$, and the forchheimer factor $(F)$. the equation shows that an inverse relationship exist between the dimensionless pressure and the Forchheimer factor. The implication of this is that the dimensionless pressure increases as the Forchheimer factor decreases. The graph below shows the effect of the Forchheimer factor on the dimensionless pressure.

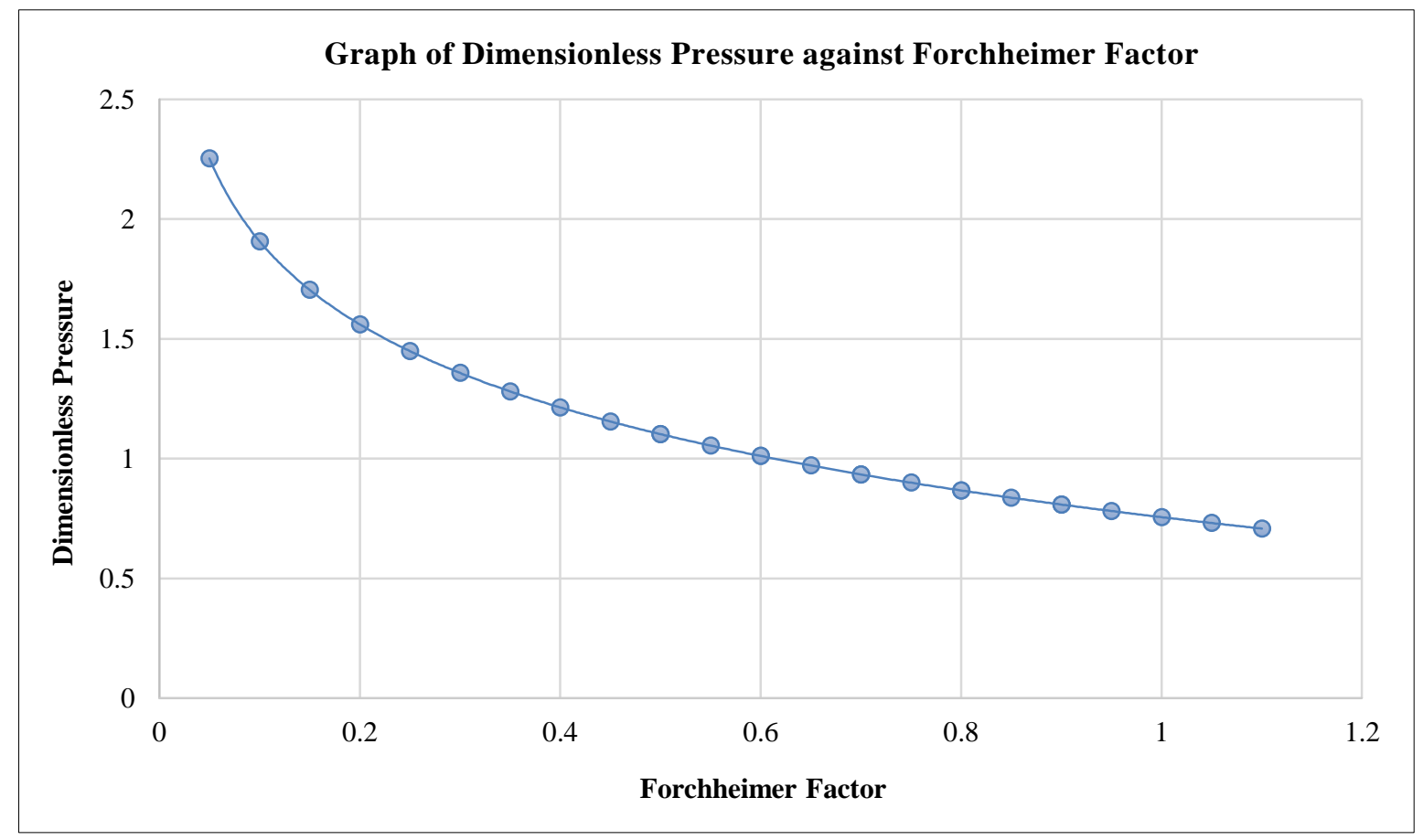

Figure 2. Effect of the Forchheimer Factor on the Dimensionless Pressure

Furthermore, Equations 20 and 21 show that the pressure drop in the reservoir has an inverse relationship with the Forchheimer factor. This implies that the pressure drop increases as the Forchheimer factor decreases. Below is the graphical representation of the relationship between pressure drop and the Forchheimer factor.

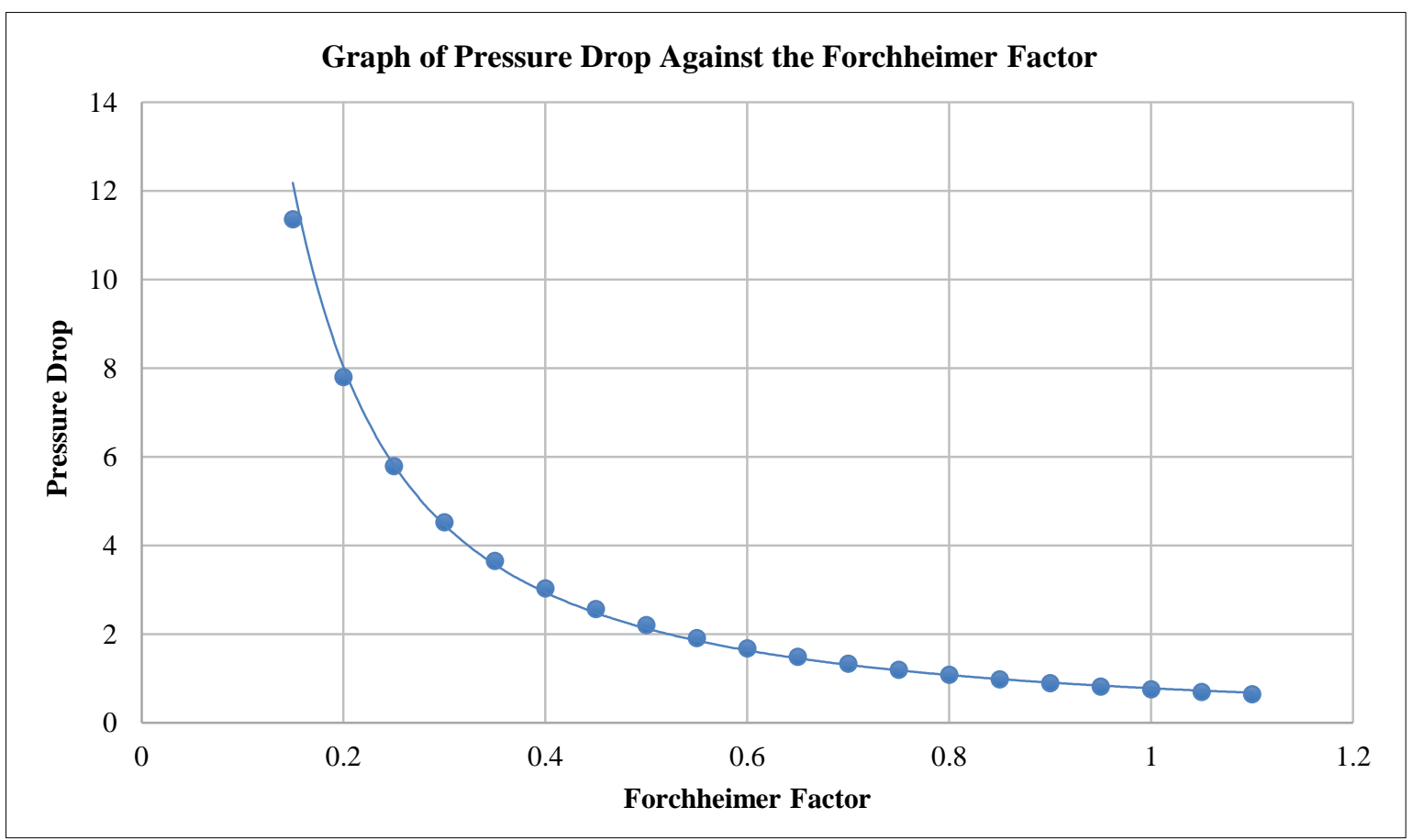

Figure 3. Effect of the Forchheimer Factor on Pressure Drop in a Reservoir 


\section{Conclusion}

A rigorous mathematical analysis of Forchheimer flow in an infinite reservoir has been carried out and simplified mathematical relationships between the parameters involved have been obtained. The significance of this study cannot be overemphasized as the Forchheimer factor has a significant effect on the instantaneous production rate, reservoir pressure, reservoir production history, and the economic analysis of hydrocarbon reservoirs. From the results above, it is evident that an inverse relationship exists between pressure drop and the Forchheimer factor. This is a result that can be applied to maximize production from a reservoir. Since pressure is the primary energy of a reservoir, minimizing the rate of pressure decline will definitely extend the production life of a reservoir while also deferring the implementation of the secondary and tertiary recovery techniques, which are costly. The results above suggest that the rate of pressure decline can be minimized by keeping the Forchheimer factor as high as possible. This can be done by deploying some engineering techniques that can help to modify the magnitudes of the reservoir and fluid properties that control the magnitude of the Forchheimer factor. Finally, it is important to note that the mathematical results presented in this paper are only applicable to homogeneous and isotropic porous media. Therefore, a more rigorous mathematical adventure must be embarked on in order to obtain similar results for heterogeneous and anisotropic porous media. Furthermore, Equations 19 and 21 must be applied with caution as we must ensure that the conditions for their validity are fully satisfied.

\section{Nomenclature}

$\begin{array}{ll}\mathrm{F}= & \text { Forchheimer factor } \\ \mu= & \text { Viscosity } \\ P_{D}= & \text { Dimensionless pressure } \\ \mathrm{P}= & \text { Pressure } \\ r_{w}= & \text { Well radius } \\ \mathrm{t}= & \text { Time } \\ \mathrm{q}= & \text { Flow rate } \\ c_{t}= & \text { Total compressibility } \\ \beta= & \text { Forchheimer coefficient }\end{array}$

$\begin{array}{ll}\Phi= & \text { Porosity } \\ \mathrm{K}= & \text { Permeability } \\ p_{i}= & \text { Initial pressure } \\ r_{D}= & \text { Dimensionless radius } \\ t_{D}= & \text { Dimensionless time } \\ \epsilon_{D}= & \text { Transformation variable } \\ \mathrm{B}= & \text { Formation volume factor } \\ \rho= & \text { Density }\end{array}$

\section{Declarations}

\subsection{Data Availability Statement}

The data presented in this study are available in article.

\subsection{Funding}

The authors received no financial support for the research, authorship, and/or publication of this article.

\subsection{Institutional Review Board Statement}

Not applicable.

\subsection{Informed Consent Statement}

Not applicable.

\subsection{Declaration of Competing Interest}

The author declares that there is no conflict of interests regarding the publication of this manuscript. In addition, the ethical issues, including plagiarism, informed consent, misconduct, data fabrication and/or falsification, double publication and/or submission, and redundancies have been completely observed by the author.

\section{References}

[1] Geertsma, J. (1974). Estimating the Coefficient of Inertial Resistance in Fluid Flow through Porous Media. Society of Petroleum Engineers of AIME Journal, 14(5), 445-450. doi:10.2118/4706-pa.

[2] Hassanizadeh, S. M., \& Gray, W. G. (1987). High velocity flow in porous media. Transport in Porous Media, 2(6), 521-531. doi:10.1007/BF00192152.

[3] Giorgi, T. (1997). Derivation of the Forchheimer Law via Matched Asymptotic Expansions. Transport in Porous Media, 29(2), 191-206. doi:10.1023/A:1006533931383.

[4] Zimmerman, R. W., Al-Yaarubi, A., Pain, C. C., \& Grattoni, C. A. (2004). Non-linear regimes of fluid flow in rock fractures. International Journal of Rock Mechanics and Mining Sciences, 41(SUPPL. 1), 163-169. doi:10.1016/j.ijrmms.2004.03.036. 
[5] Fourar, M., Radilla, G., Lenormand, R., \& Moyne, C. (2004). On the non-linear behavior of a laminar single-phase flow through two and three-dimensional porous media. Advances in Water Resources, 27(6), 669-677. doi:10.1016/j.advwatres.2004.02.021.

[6] Lucas, Y., Panfilov, M., \& Buès, M. (2007). High velocity flow through fractured and porous media: the role of flow nonperiodicity. European Journal of Mechanics, B/Fluids, 26(2), 295-303. doi:10.1016/j.euromechflu.2006.04.005.

[7] Li, J., \& Chen, C. (2020). Numerical Simulation of the Non-Darcy Flow Based on Random Fractal Micronetwork Model for Low Permeability Sandstone Gas Reservoirs. Geofluids, 2020, 9. doi:10.1155/2020/8884885.

[8] Belhaj, H. A., Agha, K. R., Nouri, A. M., Butt, S. D., \& Islam, M. R. (2003). Numerical and Experimental Modeling of NonDarcy Flow in Porous Media. Proceedings of the SPE Latin American and Caribbean Petroleum Engineering Conference, 310318. doi:10.2118/81037-ms.

[9] Wu, Y. S. (2002). Numerical simulation of single-phase and multiphase non-Darcy flow in. Transport in Porous Media, 49(2), 209-240. doi:10.1023/A:1016018020180.

[10] Dong, J., Ran, Q., Peng, H., Wang, Z., Xu, M., \& Wang, C. (2018). A Numerical Study of the Influence of Nonlinear Fluid Flow Mechanism on Dynamic Production in Tight Gas Reservoirs. Paper presented at the Abu Dhabi International Petroleum Exhibition \& Conference, Abu Dhabi, UAE, November 14, 2018. doi:10.2118/193116-ms.

[11] Nimvari, M. E., Maerefat, M., El-Hossaini, M. K., \& Jouybari, N. F. (2014). Numerical study on turbulence effects in porous burners. Journal of Porous Media, 17(2), 129-142. doi:10.1615/JPorMedia.v17.i2.40.

[12] Wen, Z., \& Wang, Q. (2013). Approximate analytical and numerical solutions for radial non-Darcian flow to a well in a leaky aquifer with wellbore storage and skin effect. International Journal for Numerical and Analytical Methods in Geomechanics, 37(11), 1453-1469. doi:10.1002/nag.2091.

[13] Wen, Z., Huang, G., \& Zhan, H. (2009). A numerical solution for non-Darcian flow to a well in a confined aquifer using the power law function. Journal of Hydrology, 364(1-2), 99-106. doi:10.1016/j.jhydrol.2008.10.009.

[14] Adeyemi, T. S., \& Rufus, D. O. (2021). Analytical Development of an Improved Inflow Performance Relationship (IPR) Model for Solution Gas Drive Reservoirs. Journal of Human, Earth, and Future, 2(2), 125-135. doi:10.28991/hef-2021-02-0204 .

[15] Lucas, Y., Panfilov, M., \& Buès, M. (2008). The impact of instability appearance on the quadratic law for flow through porous media. Transport in Porous Media, 71(1), 99-113. doi:10.1007/s11242-007-9113-8.

[16] Ma, H., \& Ruth, D. W. (1993). The microscopic analysis of high Forchheimer number flow in porous media. Transport in Porous Media, 13(2), 139-160. doi:10.1007/BF00654407.

[17] Mathias, S. A., Butler, A. P., \& Zhan, H. (2008). Approximate Solutions for Forchheimer Flow to a Well. Journal of Hydraulic Engineering, 134(9), 1318-1325. doi:10.1061/(asce)0733-9429(2008)134:9(1318).

[18] Moutsopoulos, K. N., \& Tsihrintzis, V. A. (2005). Approximate analytical solutions of the Forchheimer equation. Journal of Hydrology, 309(1-4), 93-103. doi:10.1016/j.jhydrol.2004.11.014.

[19] Ojo Ibukun Joel, A. T. S. (2021). a Novel Approach To Forecasting Production Rate of Dry Gas Wells. International Journal of Innovations in Engineering Research and Technology, 6(11), 1-6.

[20] Sedghi-Asl, M., Rahimi, H., \& Salehi, R. (2014). Non-Darcy Flow of Water through a Packed Column Test. Transport in Porous Media, 101(2), 215-227. doi:10.1007/s11242-013-0240-0.

[21] Wen, Z., Huang, G., \& Zhan, H. (2011). Non-Darcian flow to a well in a leaky aquifer using the Forchheimer equation. Hydrogeology Journal, 19(3), 563-572. doi:10.1007/s10040-011-0709-2.

[22] Panfilov, M., \& Fourar, M. (2006). Physical splitting of nonlinear effects in high-velocity stable flow through porous media. Advances in Water Resources, 29(1), 30-41. doi:10.1016/j.advwatres.2005.05.002.

[23] Sheriff, A. T. (2018). Application of the linear flow diffusivity equation in estimating water influx in linear water drive reservoirs. IOJPH - International Open Journal of Applied Science, 1(3), 12-25.

[24] Wen, Z., Liu, K., \& Chen, X. (2013). Approximate analytical solution for non-Darcian flow toward a partially penetrating well in a confined aquifer. Journal of Hydrology, 498, 124-131. doi:10.1016/j.jhydrol.2013.06.027.

[25] Zeng, F., \& Zhao, G. (2008). Semianalytical model for reservoirs with Forchheimer's non-Darcy flow. SPE Reservoir Evaluation and Engineering, 11(2), 280-291. doi:10.2118/100540-pa. 